\title{
Dielectric Confinement Enables Molecular Coupling in Stacked Colloidal Nanoplatelets
}

\author{
José L. Movilla ${ }^{\dagger}$ Josep Planelles, ${ }^{\ddagger}$ and Juan I. Climente, ${ }^{*} \ddagger$ \\ †Dept. d'Educació i Didàctiques Específiques, Universitat Jaume I, 12080, Castelló, Spain \\ $\ddagger$ Departament de Química Física i Analítica, Universitat Jaume I, E-12080, Castelló de la \\ Plana, Spain \\ E-mail: climente@uji.es
}

\begin{abstract}
We show theoretically that carriers confined in semiconductor colloidal nanoplatelets (NPLs) sense the presence of neighbor, cofacially stacked NPLs in their energy spectrum. When approaching identical NPLs, the otherwise degenerate energy levels redshift and split, forming (for large stacks) minibands of several meV width. Unlike in epitaxial structures, the molecular behavior does not result from quantum tunneling but from changes in the dielectric confinement. The associated excitonic absorption spectrum shows a rich structure of bright and dark states, whose optical activity and multiplicity can be understood from reflection symmetry and Coulomb tunneling. We predict spectroscopic signatures which should confirm the formation of molecular states, whose practical realization would pave the way to the development of nanocrystal chemistry based on NPLs.
\end{abstract}

\section{Keywords}

colloidal nanocrystal, nanoplatelet, superlattice, dielectric mismatch, exciton 
Semiconductor quantum dots synthesized via wet chemistry, also known as colloidal nanocrystals, have reached a level of maturity that makes them suitable for existing and emerging optical and electronic technologies. ${ }^{1}$ A natural step for further progress is the controlled coupling of nanocrystals, by which the atomic-like energy levels of the dots interact with those of neighbors to form a molecular-like spectrum. Current endeavor to develop this so-called "nanocrystal chemistry" largely relies on the design of coupled nanocrystal structures, from dimers ${ }^{2,3}$ to superlattices, ${ }^{4,5}$ where quantum tunneling of charged carriers ${ }^{6}$ (electrons, holes) or dipole-dipole interactions of neutral carriers ${ }^{7-9}$ (excitons) are exploited.

As a newcomer in the family of nanocrystals, colloidal NPLs have attracted much interest in the last years. ${ }^{10,11}$ Their quasi-two-dimensional structure with precise (atomic monolayer) thickness control results in extremely bright and narrow fluorescent emission, which makes them particularly promising for optoelectronic applications. ${ }^{1,8,10-13}$ On the other hand, the potential of NPLs as building blocks for molecular superstructures with electronic coupling is still unclear. Van der Waals attraction enables the self-assembly of cofacially stacked NPLs, where the inorganic semiconductor alternates with few-nm-thick layers of organic ligands. ${ }^{14,15}$ The stacked NPLs can be chosen to have identical thickness, thus constituting a nearly ideal homonuclear molecule, with negligible size polydispersity. However, the formation of electron or hole energy minibands driven by quantum tunneling -analogous to those in epitaxial quantum well superlattices ${ }^{16}$ - is inhibited because the ligands typically impose a high $(2-4 \mathrm{eV})$ potential barrier. The constitution of such energy minibands would be of great practical interest, as an additional degreee of freedom in the electronic structure design and to possibly combine the outstanding optical properties of individual NPLs with transversal transport properties of superlattices.

Recently, low temperature photoluminescence measurements of CdSe NPLs have revealed two well resolved emission peaks instead of one. ${ }^{17-21}$ Diroll and co-workers showed that the relative intensity and energy splitting of the two peaks depend on the degree of stacking and inter-NPL distance, respectively. ${ }^{21}$ These observations suggest that inter-NPL excitations 
take place in stacked NPLs. With quantum tunneling quenched by the organic spacer layer, the fundamental question arises of what the origin of such interactions is, and how can they be controlled.

The goal of this work is to provide a theoretical framework to answer the above questions. We calculate the electronic structure of electrons, holes and excitons in stacked NPLs. We shall see that molecular interactions are in fact enabled by the drastically different polarizability of NPLs and ligands, which make self-energy and Coulomb screening terms sensitive to the number, position and proximity of nearby NPLs. Beyond assessing on Ref. ${ }^{21}$ observations, our results provide a general picture of how inter-NPL interactions operate in colloidal systems and what experimental signatures should be sought in future experiments to confirm the formation of molecular states driven by dielectric confinement.

Charged carriers are described with effective mass Hamiltonians,

$$
H^{j}=\frac{\mathbf{p}_{\perp}{ }^{2}}{2 m_{\perp}^{j}}+\frac{p_{z}^{2}}{2 m_{z}^{j}}+V_{b o}^{j}\left(\mathbf{r}_{\mathbf{j}}\right)+\Sigma\left(\mathbf{r}_{\mathbf{j}}\right) .
$$

where $j=e, h$ stands for electron and hole, respectively. $m_{z}^{j}\left(m_{\perp}^{j}\right)$ is the mass parallel (perpendicular) to the strong confinement direction of the NPL, $V_{b o}^{j}$ is the potential bandoffset between the NPL and the organic environment, and $E_{\Sigma}$ the self-energy potential arising from the dielectric mismatch between the two materials. ${ }^{22-25}$

Since the ratio of NPL/ligand dielectric constants fulfills $\epsilon_{\text {in }} / \epsilon_{\text {out }}>1$, a charge inside the NPL creates a surface polarization field of the same (opposite) sign on the inner (outer) side of the NPL. ${ }^{22,23} \Sigma$ is then repulsive inside the NPL and attractive in between NPLs. From this basic consideration, we can foresee the qualitative electronic structure of stacked NPLs, which is shown in Figure 1(a). For a single NPL (left part of figure), assuming infinite potential barrier, the electron (or hole) ground state energy is given by $E_{\text {conf }}+E_{\Sigma}$. The former is the quantum confinement energy and the latter the correction coming from selfenergy repulsion. When two NPLs are considered, since tunneling is largely suppressed in 

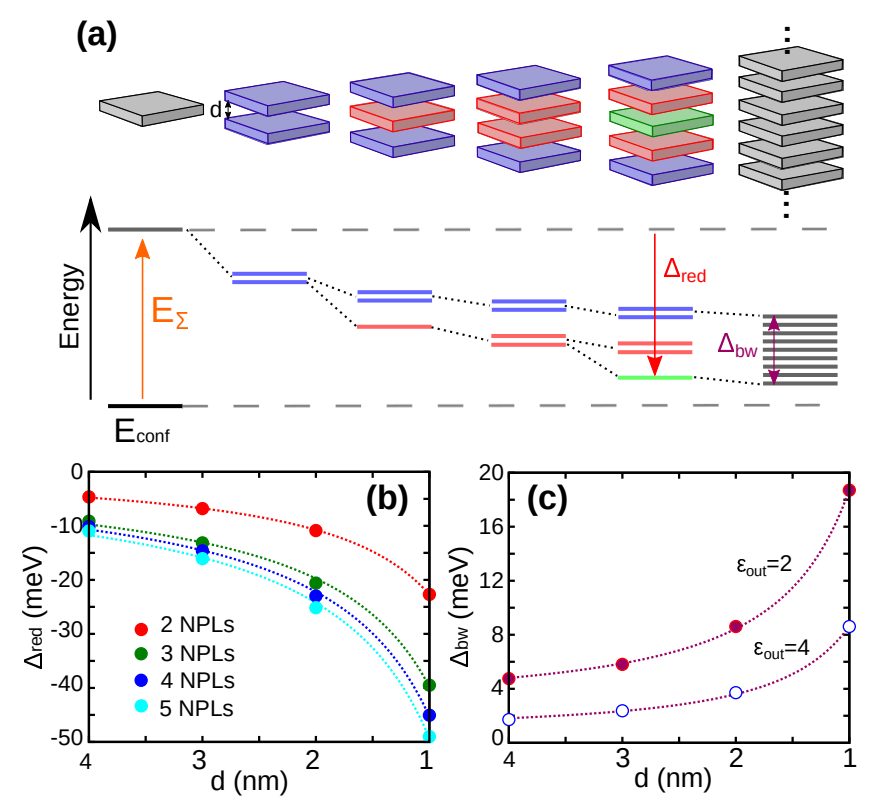

Figure 1: (a) Schematic energy spectrum of a single particle in stacks of NPLs. With increasing number of NPLs, $\Sigma$ gradually weakens, the energy levels redshift and degeneracy is lifted. Minibands are expected for periodic arrays of NPLs. (b) Ground state redshift for different number of NPLs, as a function of the inter-NPL distance. Dots are calculated values, lines $1 / d$ fits. (c) Band width of a 5 NPL stack, for two outer dielectric constants. Dots are calculated values, lines $1 / d^{3 / 2}$ fits.

colloidal systems, quantum confinement energy is again $E_{\text {conf }}$. However, if the inter-NPL distance $d$ is short enough, a charge confined inside a NPL polarizes not only the host NPL but also the neighbor one. The dielectric confinement is then weaker than for isolated NPLs, and $E_{\Sigma}$ decreases. This gives rise to a redshift of the (doubly degenerate) ground state. For three NPLs, the same reasoning implies the redshift further increases. Moreover, in this case the redshift is more pronounced for the central platelet than for the terminal ones, because two nearby (first) neighbors weaken dielectric confinement more efficiently than a first and a second neighbor. This asymmetry of dielectric environment splits the otherwise triply degenerate energy levels into a singlet (central NPL) and a doublet (two terminal NPLs). Similar trends hold for increasing number of NPLs, but the redshift saturates because the influence of distant NPLs vanishes. At the same time, the number of states keeps on increasing. As a result, the electronic structure evolves towards the formation of an energy miniband, reminiscent of that in quantum well superlattices, but driven by self-energy instead 
of tunneling.

The central question about the scenario pictured in Fig. 1(a) is whether the magnitudes of the redshift $\left(\Delta_{r e d}\right)$ and band width $\left(\Delta_{b w}\right)$ is of practical significance. To address this point, we solve Hamiltonian (1) for a conduction band electron in a stack of 4.5-monolayers $(1.35 \mathrm{~nm})$ thick CdSe NPLs with lateral side $L=10 \mathrm{~nm}$ (see Supporting Information, SI, for details). Dielectric constants $\epsilon_{i n}=10$ and $\epsilon_{\text {out }}=2$ are taken, which are typical values for the CdSe NPL/ligand system. ${ }^{25,26}$ Usual analytical expressions to calculate $\Sigma$, based on the method of image charges,${ }^{27}$ become impractical in stacks. We then calculate numerically the surface induced charge using Ref. ${ }^{28}$ algorithms and codes. The ensuing $\Sigma$ compares very well with image charge results for a single quantum well (Fig. S2 in SI), and provides a valid extension for coupled quantum wells. Representations of $\Sigma$ along the $z$ axis of stacks of NPLs are shown in Figs. S3 and S4. The self-energy potential is found to be quasi-additive down to small inter-NPL distances values, i.e. it is roughly the superimposition of $\Sigma$ for two independent NPLs separated by $d$. This validates the reasoning used to deduce Fig. 1(a), and in particular the expected stabilization of the central NPLs as compared to terminal ones.

Figs. 1(b) and 1(c) (solid dots) show the magnitude of $\Delta_{r e d}$ and $\Delta_{b w}$ as NPLs are brought together. Both magnitudes increase rapidly, following $1 / d$ and $1 / d^{3 / 2}$ scaling laws, respectively. For a oleic acid $\left(d \approx 4 \mathrm{~nm}^{14}\right)$ they are in the $4-10 \mathrm{meV}$ range, but reach few tens of meV for shorter ligands $(d \approx 1 \mathrm{~nm})$. We stress that this energy scale is in fact comparable to that of minibands in epitaxial quantum well superlattices, ${ }^{16}$ and large enough to be experimentally observable e.g. through tunneling spectroscopy ${ }^{29}$ On a more conservative estimate, we set the ligand dielectric constant to $\epsilon_{\text {out }}=4$. Because $\Sigma \propto\left(\epsilon_{\text {in }}-\epsilon_{\text {out }}\right) /\left(\epsilon_{\text {in }}+\epsilon_{\text {out }}\right){ }^{22,23}$ the energy splittings are reduced, but they are still sizable for short distances. One example is shown in Fig. 1(c), empty dots.

To investigate the optical signatures resulting from the electronic structure of Fig. 1(a), we first plot the absorption of an electron-hole (e-h) pair, disregarding Coulomb interaction. 

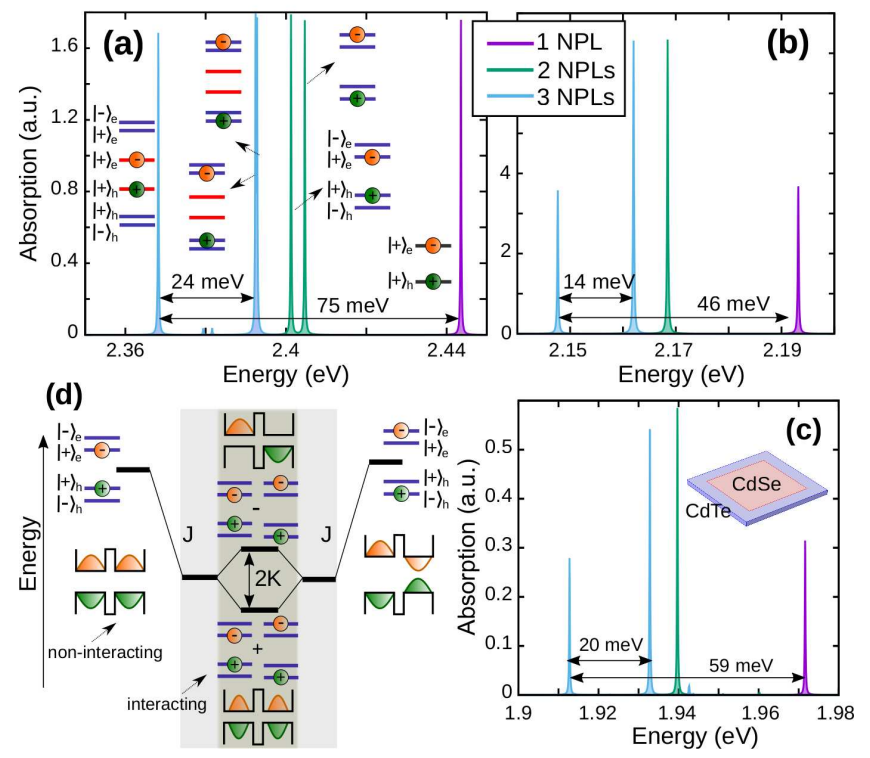

Figure 2: Absorption spectrum in stacks of 1, 2 and 3 NPLs with inter-NPL distance $d=1$ nm. (a) e-h pair, disregarding Coulomb interaction. The insets show the electronic configuration of the bright states. (b) Same but including e-h interaction (exciton). (c) Exciton in a CdSe/CdTe core/crown NPL. The core (crown) side size is $L=10 \mathrm{~nm}(L=14 \mathrm{~nm})$. (d) Schematic of the effect of Coulomb interaction. Nearly-degenerate e-h states are stabilized by $J$ and split by Coulomb tunneling to form orthogonal (direct and indirect) exciton states.

For convenience of presentation, we choose a small inter-NPL separation $(d=1 \mathrm{~nm})$, which enables a small amount of quantum tunneling. Fig. 2(a) shows the spectrum calculated for stacks of 1, 2 and 3 NPLs. A number of relevant features are observed: (i) the peaks redshift as the number of NPLs increases, (ii) the number of peaks equals the number of stacked NPLs, ${ }^{30}$ (iii) the peaks of a given stack are split energetically. Feature (i) is the consequence of the self-energy weakening in stacked NPLs, through $\Delta_{\text {red }}$. The redshift is about twice larger than in Fig.1(b) for the same $d$, because the self-energies of electron and hole add up. Feature (ii) follows from selection rules. Electron and hole states can be classified by their parity (symmetry with respect to a horizontal reflection plane in the center of the stack), $\left|\sigma_{j}\right\rangle=|+\rangle$ or $|-\rangle$. For the e-h pair, only states with total parity even $\left(\left|\sigma_{e h}\right\rangle=\left|\sigma_{e}\right\rangle \times\left|\sigma_{h}\right\rangle=\right.$ $|+\rangle)$ are bright. Otherwise, electron and hole have different symmetry and their overlap is zero. Considering one level per NPL, and the fact that parity sign alternates with increasing energy, the only optically bright configurations are those shown in the insets of Fig. 2(a). As 
for feature (iii), the origin of the energy splittings can be understood from the configurations. For 2 NPLs, the two peaks correspond to bonding and antibonding molecular orbitals split by tunneling energy. The splitting is small $(3 \mathrm{meV})$ in spite of the short inter-NPL distance $(d=1 \mathrm{~nm})$ because the ligands constitute a high potential barrier. For 3 NPLs, the second and third peak (nearly degenerate) again correspond to bonding and antibonding states localized in the terminal NPLs. Tunnel splitting is also present, but it is negligible because the distance between terminal NPLs is $2 d+L_{z}$, where $L_{z}$ is the thickness of the central NPL. The red-most peak in turn originates in the central platelet. It shows a remarkably large stabilization $(24 \mathrm{meV}$ ) as compared to the peaks of terminal NPLs, this being a signature of the reduced $\Sigma$.

To analyze the effect of adding e-h Coulomb interaction, we next compute exciton states from the Hamiltonian $H_{X}=H_{e}+H_{h}+V_{e h}$. Here $V_{e h}=V_{e h}^{0}+V_{e h}^{p o l}$ is the e-h Coulomb attraction, with $V_{e h}^{0}$ describing the locally screened interaction and $V_{e h}^{p o l}$ the interaction of one charge with the surface polarization created by the other one (Coulomb polarization term). ${ }^{22,23}$ Eigenstates are obtained with a full configuration interaction calculation on the basis of Hartree products of single-particle e-h spin-orbitals, built out of $s, p_{x}$ and $p_{y}$ orbitals for each NPL of the stack. This basis set accounts for the core contribution of both vertical and in-plane electronic correlations. The latter have been shown to be important in type-I NPLs. ${ }^{31,32}$ Fig.2(b) shows the resulting absorption spectrum. As compared to Fig. 2(a), a few relevant differences appear: (i) the whole spectrum is shifted to the red by $\sim 200 \mathrm{meV}$, (ii) the interpeak splittings have shrinked (e.g. from 24 to $14 \mathrm{meV}$, in the case of $3 \mathrm{NPLs}$ ) and (iii) some peaks vanish, e.g. the second peak of the 2 NPL stack. The first difference is a consequence of the strong exciton binding energy. ${ }^{12,25,26,31}$ The second difference is because the stronger dielectric confinement of terminal NPLs (as compared to central ones) not only enhances $\Sigma$, but also $V_{e h}^{p o l}$. Because the two terms have opposite signs (repulsive vs attractive), there is a partial cancellation. It is worth noting that the cancellation is exact to first order of perturbation in strongly confined, spherical nanocrystals. ${ }^{33}$ For this 
reason, energetic signatures of dielectric confinement are often negligible in superlattices of quantum dot nanocrystals. ${ }^{5,34}$ However, in anistropic NPLs with weak lateral confinement the compensation is far from exact, with self-energy terms prevailing. ${ }^{25,35,36}$ This makes colloidal NPL systems particularly suitable to tune the exciton energy through dielectric confinement. In addition, the compensation can be further reduced by resorting to type-II structures. Fig.2(c) shows the exciton absorption in stacks of core-crown CdSe/CdTe NPL. Here the electron stays in the CdSe core, while the hole localizes in the CdTe crown. $V_{e h}$ is then reduced and, consequently, the energy splittings between peaks increase as compared to CdSe core-only NPLs of Fig. 2(b).

The missing peaks in Fig. 2(b), difference (iii), are a consequence of Coulomb tunneling. A non-interacting e-h pair inside a pair of dielectrically equivalent NPLs gives two optically bright states, $|+\rangle_{e}|+\rangle_{h}$ and $|-\rangle_{e}|-\rangle_{h}$, possibly split by a small quantum tunneling energy (see e.g. the case of 2 NPLs in Fig. 2(a)). Upon inclusion of Coulomb interaction, both states benefit from a direct term, $J=\left\langle+\left.\right|_{e}\left\langle+\left|{ }_{h} V_{e h}\right|+\right\rangle_{e} \mid+\right\rangle_{h} \approx\left\langle-\left.\right|_{e}\left\langle-\left.\right|_{h} V_{e h} \mid-\right\rangle_{e} \mid-\right\rangle_{h}$, but because they share the same total parity they are further admixed by a crossed term, $K=\left\langle+\left.\right|_{e}\left\langle+{ }_{h} V_{e h} \mid-\right\rangle_{e} \mid-\right\rangle_{h}$. The resulting exciton eigenfunctions are the symmetric and antisymmetric linear combinations, $\left|\sigma_{e h}\right\rangle_{ \pm}=|+\rangle_{ \pm}=1 / \sqrt{2}\left(|+\rangle_{e}|+\rangle_{h} \pm|-\rangle_{e}|-\rangle_{h}\right)$, which have Coulomb expectation values $\left\langle V_{e h}\right\rangle=J \pm K$. The whole interaction scheme is depicted in Fig. 2(d). It is easy to show that $\left|\sigma_{e h}\right\rangle_{+}$corresponds to a direct exciton, where the hole stays in the same NPL as the electron, and $\left|\sigma_{e h}\right\rangle_{-}$to the indirect one, where the two carriers avoid each other (see insets in Fig. 2(d)). The latter is dark because e-h overlap vanishes, which explains the missing peaks of Fig. 2(b). The crossed interaction $K$ is a Coulomb tunneling term, akin to quantum tunneling in that it splits two otherwise quasi-degenerate states in two nearby nanostructures. It is then a molecular interaction, but acting on excitons instead of single carriers. It reflects the stabilization (destabilization) when the e-h pair is forced to localize in the same (opposite) NPL. Coulomb and quantum tunneling are however competitive processes, because the former suppresses wave function delocalization in between NPLs. 
Contrary to epitaxial heterostructures, where both terms can be comparable, ${ }^{37}$ in colloidal nanocrystals Coulomb tunneling is largely dominant (see Figs. S6 and S7 in SI).

(a)
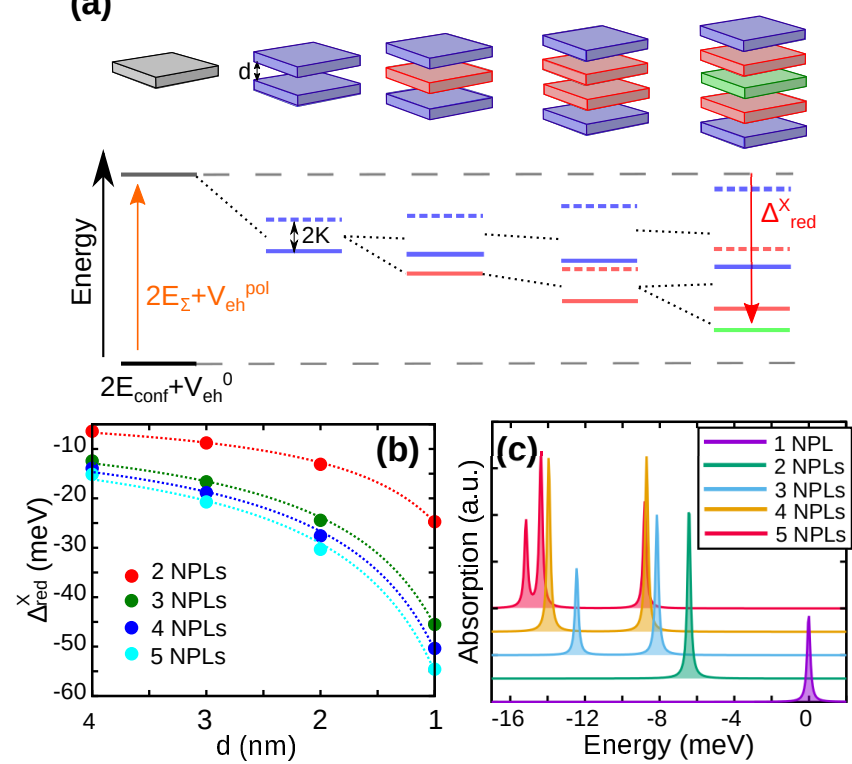

Figure 3: (a) Schematic energy spectrum of excitons with $\left|\sigma_{e h}\right\rangle=|+\rangle$ in stacks of NPLs. Solid (dashed) lines are direct (indirect) excitons. (b) Exciton ground state redshift as a function of the inter-NPL distance. Dots are calculated values, lines $1 / d$ fits. (c) Exciton absorption spectrum for $d=4 \mathrm{~nm}$, centered at the energy of one isolated NPL. The number of peaks relates to the number of stacked NPLs as explained in panel (a).

Gathering single particle $(\Sigma)$ and two-body $\left(V_{e h}^{p o l}\right)$ effects of dielectric confinement, we can portrait the energy structure of excitons in stacks of NPLs. Fig. 3(a) represents a qualitative schematic, displaying exciton states with $\left|\sigma_{e h}\right\rangle=|+\rangle$. Similar to the single particle case, with increasing number of NPLs the states tend to redshift and split owing to the weakened dielectric terms, $\left(2 \Sigma+V_{e h}^{p o l}\right)$. In addition, the doublets coming from a pair of equivalent NPLs split to form direct and indirect excitons, $|+\rangle_{ \pm}$, split by $2 K$. Only direct exciton states remain bright. Consequently, the exciton absorption shows one bright exciton per each type of dielectrically equivalent NPLs, solid lines in Fig. 3(a). This explains why the absorption spectrum, Fig. 3(c), shows a single peak for 1 or 2 NPLs, two for 3 or 4 NPLs, and three for 5 NPLs (one for central platelet, one for terminal ones, one for intermediate ones). The same rule holds for larger number of NPLs, but the energy splittings are increasingly small. 
Fig. 3(b) shows the exciton ground state redshift $\left(\Delta_{\text {red }}^{X}\right)$ as a function of the inter-NPL distance, for CdSe NPLs. As in the single-particle case, numerical estimates are well fit by a $1 / d$ scaling. The overall magnitude of $\Delta_{\text {red }}^{X}$ is similar to that of Fig. $1(\mathrm{~b})$, in spite of having two particles adding their self-energies. This indicates that $V_{e h}^{p o l}$ roughly compensates for one $\Sigma$ term.

Altogether Fig. 3 points that it should be possible to find optical signatures of molecular coupling in stacked NPLs, in the form of energetically resolved multiplets of optically active peaks, the number of such peaks reflecting the types of dielectrically equivalent NPLs in the stack. It is worth stressing that no such signatures are expected in the absence of dielectric confinement. The exciton absorption spectrum shows then a single peak, whose energy is independent of the number of stacked NPLs, see Fig. S5.

One can try establish connections between the results in Fig. 3 and the specific experiments of Ref., ${ }^{21}$ which showed that low-temperature emission of ensembles of presumably stacked CdSe NPLs depends on $d$. From our theory, the exciton ground state peak of an isolated NPLs is clearly blueshifted when compared to that of stacked NPLs (see Figs. 3(b) and (c)). This offers a possible explanation for the two-color emission observed in the experiments, whereby the high-energy peak comes from isolated NPLs and the low-energy (broader) one from stacks with variable number of NPLs. Different experimental features are then explained, such as the energy splitting scaling inversely with $d$, or the fact that the low-energy peak red-shifts and gains intensity as the degree of stacking increases (e.g. through addition of ethanol). However, the magnitude of the experimental splitting, $20-30$ meV, requires $d=2 \mathrm{~nm}$ in our simulations, about half the reported inter-NPL length with oleic acid. ${ }^{14,21}$ This seems unlikely. The double peak feature might still arise from exciton vs. trion emission. ${ }^{19,20}$ The $d$-dependence could then be explained from the different weight of $\Sigma$ and $V_{e h}^{p o l}$ in exciton (neutral charge) and trion (net charge) species. Beyond NPLs, the dielectric effects we predict when colloidal nanostructures are approached provide a potential interpretation for the emission redshift recently observed in superlattices of $\mathrm{CsPbBr}_{3}$ and 
charged CdTe nanocrystals, where quantum tunneling is not expected. ${ }^{38,39}$

Further experiments are now needed to confirm the molecular coupling predicted by theory. Vertically stacked NPLs constitute an optimal system to this end because the reduced yet precisely controlled thickness permits small spacing between neighbour, isoenergetic structures. The strongly anistropic geometry of individual NPLs also reduces compensations between $\Sigma$ and $V_{e h}^{p o l}$, thus favoring optical manifestations. Other ideal experimental conditions involve short ligands to minimize $d$, yet posing a large dielectric mismatch with the inorganic NPLs. Large NPL lateral dimensions and reduced interlayer misorientation are convenient to favor degeneracy between stacked NPLs. The use of type-II hetero-NPLs to reduce the compensation of $\Sigma$ and $V_{e h}^{p o l}$ is also benefitial, even though the irregular core-shell interface may constitute a source of linewidth broadening. Low temperature absorption is desirable to avoid phonon-broadening ${ }^{40}$ and Forster resonant energy transfer towards defective platelets, ${ }^{9}$ which could impair the observation of fine structure molecular effects. Likewise, single particle (optical or transport) spectroscopy is likely needed to avoid convoluted signals coming from stacks of different length. Verifying electronic coupling between NPLs would not only prove a novel form of molecular interaction between nanocrystals, but also path the way to the development of NPL superstructures whose collective properties differ from those of individual components.

In conclusion, we have shown that the electronic structure of electrons, holes and excitons in colloidal NPLs can be modified through stacking. Carriers confined in a NPL polarize the nearby NPLs, altering the dielectric environment. This constitutes a form of molecular coupling, where the energy levels of individual components split under the effect of selfenergy, Coulomb polarization and Coulomb tunneling terms. For large stacks, this leads to the formation of minibands with potential band width of several meV, whose practical realization would add a degree of freedom in the design of the electronic structure, and possibly combine the excellent optical properties of NPLs with the improved transversal mobility of quantum well superlattices. 


\section{Associated Content}

Supporting Information. Includes (i) additional details on theoretical model, including material parameters and self-energy potential profiles in stacked NPLs, (ii) supporting calculations on the role of dielectric mismatch and Coulomb tunneling in defining the optical spectrum of excitons, and wave function localization.

\section{Acknowledgement}

We acknowledge support from MICINN project CTQ2017-83781-P and UJI project B201759.

\section{References}

(1) M. V. Kovalenko, L. Manna, A. Cabot, Z. Hens, D. V. Talapin, C. R. Kagan, V. I. Klimov, A. L. Rogach, P. Reiss, D. J. Milliron, P. Guyot-Sionnest, G. Konstantatos, W. J. Parak, T. Hyeon, B. A. Korgel, C. B. Murray, and W. Heiss, "Prospects of Nanoscience with Nanocrystals", ACS Nano 9, 1012 (2015).

(2) A. Teitelboim, N. Meir, M. Kazes, and D. Oron, "Colloidal Double Quantum Dots", Acc. Chem. Res. 49, 902 (2016).

(3) J. Cui, Y. E. Panfil, S. Koley, D. Shamalia, N. Waiskopf, S. Remennik, I. Popov, M. Oded, and U. Banin, "Coupled Colloidal Quantum Dot Molecules", Nat. Commun. 10, 5401 (2019).

(4) M. A. Boles, M. Engel, and D. V. Talapin, "Self-Assembly of Colloidal Nanocrystals: From Intrincate Structures to Functional Materials", Chem. Rev. 116, 112220 (2016).

(5) K. Whitham, J. Yang, B. H. Savitzky, L. F. Kourkoutis, F. Wise, and T. Hanrath, 
"Charge transport and localization in atomically coherent quantum dot solids", Nature Mater. 15, 557 (2016).

(6) A. Tadjine, and C. Delerue, "Colloidal nanocrystals as LEGO (R) bricks for building electronic band structure models", Phys. Chem. Chem. Phys. 20, 8177 (2018).

(7) S. A. Crooker, J. A. Hollingsworth, S. Tretiak, and V. I. Klimov, "Spectrally Resolved Dynamics of Energy Transfer in Quantum-Dot Assemblies: Towards Engineered Energy Flows in Artificial Materials", Phys. Rev. Lett. 89, 186802 (2002).

(8) C. E. Rowland, I. Fedin, H. Zhang, S. K. Gray, A. O. Govorov, D. V. Talapin, and R. D. Schaller, "Picosecond energy transfer and multiexciton transfer outpaces Auger recombination in binary CdSe nanoplatelet solids", Nature Mater. 14, 484 (2015).

(9) B. Guzelturk, O. Erdem, M. Olutas, Y. Kelestemur, H. Volkan Demir, "Stacking in Colloidal Nanoplatelets: Tuning Excitonic Properties", ACS Nano 8, 12524 (2014).

(10) E. Lhuillier, S. Pedetti, S. Ithurria, B. Nadal, H. Heuclin, and B. Dubertret, "TwoDimensional Colloidal Metal Chalcogenides Semiconductors: Synthesis, Spectroscopy, and Applications", Acc. Chem. Res. 48, 22 (2015).

(11) P. Tyagi, S. M. Arveson, and W. A. Tisdale, "Colloidal Organohalide Perovskite Nanoplatelets Exhibiting Quantum Confinement", J. Phys. Chem. Lett. 6, 1911 (2015).

(12) J.Q. Grim, S. Christodoulou, F. Di Stasio, R. Krahne, R. Cingolani, L. Manna, and I. Moreels, "Continuous-wave biexciton lasing at room temperature using solutionprocessed quantum wells", Nature Nanotech. 9, 891 (2014).

(13) R. Scott, J. Heckmann, A.V. Prudnikau, A. Antanovich, A. Mikhailov, N. Owschimikow, M. Artemyev, J.I. Climente, U. Woggon, N.B. Grosse, and A.W. Achtstein, "Directed emission of CdSe nanoplatelets originating from strongly anisotropic 2D electronic structure", Nature Nanotech. 12, 1155 (2017). 
(14) S. Jana, T. N. T. Phan, C. Bouet, M. D. Tessier, P. Davidson, B. Dubertret, and B. Abecassis, "Stacking and Colloidal Stability of CdSe Nanoplatelets", Langmuir 31, 10532 (2015).

(15) K.J. Lee, B. Turedi, L. Sinatra, A.A. Zhumekenov, P. Maity, I. Dursun, R. Naphade, N. Merdad, A. Alsalloum, S. Oh, N. Wehb, M.N. Hedhili, C. H. Kang, R. C. Subedi, N. Cho, J. S. Kim, B. S. Ooi, O. F. Mohammed, and O. M. Bakr, "Perovskite-Based Artificial Multiple Quantum Wells", Nano Lett 19, 3535 (2019).

(16) E. E. Mendez, K. von Klitzing (eds), Physics and Applications of Quantum Wells and Superlattices, (Nato Science Series B, 1987).

(17) M. D. Tessier, L. Biadala, C. Bouet, S. Ithurria, B. Abecassis, and B. Dubertret, "Phonon Line Emission Revealed by Self-Assembly of Colloidal Nanoplatetets", ACS Nano 7, 3332-3340 (2013).

(18) A. W. Achtstein, R. Scott, S. Kickhöfel, S. T. Jagsch, S. Christodoulou, G. H. V. Bertrand, A. V. Prudnikau, A. Antanovich, M. Artemyev, I. Moreels, A. Schliwa and U. Woggon, "p-State Luminescence in CdSe Nanoplatelets, The Role of Lateral Confinement and a Longitudinal Optical Phonon Bottleneck", Phys. Rev. Lett. 116, 116802 (2016).

(19) J. Yu, C. Zhang, G. Pang, X. Wei Sun and R. Chen, "Effect of Lateral Size and Surface Passivation on the Near-Band-Edge Excitonic Emission from Quasi-Two-Dimensional CdSe Nanoplatelets", ACS Appl. Mater. Interfaces 11 41821-41827 (2019).

(20) E V. Shornikova, D. R. Yakovlev, L. Biadala, S. A. Crooker, V. V. Belykh, M. V. Kochiev, A. Kuntzmann, M. Nasilowski, B. Dubertret and M. Bayer "Negatively Charged Excitons in CdSe Nanoplatelets", Nano Lett 20, https://doi.org/10.1021/acs.nanolett.9b04907 
(21) B. T. Diroll, W. Cho, I. Coropceanu, S. M. Harvey, A. Brumberg, N. Holtgrewe, S. A. Crooker, M. R. Wasielewski, V. B. Prakapenka, D. V. Talapin, and R. D. Schaller, "Semiconductor Nanoplatelet Excimers", Nano Lett 18, 6948 (2018).

(22) A. V. Rodina, Al. L. Efros, "Effect of dielectric confinement on optical properties of colloidal nanostructures", J. Exp. Theo. Phys. 122, 554 (2016).

(23) C. Delerue, and M. Lannoo, Nanostructures: Theory and Modelling, (Springer-Verlag, Berlin, 2004).

(24) J. Even, L. Pedesseau, and M. Kepenekian, "Electronic surface states and dielectric self-energy profiles in colloidal nanoscale platelets of CdSe", Phys. Chem. Chem. Phys. 16, $25182(2014)$.

(25) R. Benchamekh, N. A. Gippius, J. Even, M. O. Nestoklon, J. M. Jancu, S. Ithurria, B. Dubertret, Al. L. Efros, and P. Voisin, "Tight-binding calculations of image charge effects in colloidal nanoscale platelets of CdSe", Phys. Rev. B 89, 035307 (2014).

(26) A.W. Achtstein, A. Schliwa, A. Prudnikau, M. Hardzei, M.V. Artemyev, C. Thomsen, and U. Woggon, "Electronic Structure and Exciton-Phonon Interaction in TwoDimensional Colloidal CdSe Nanosheets", Nano Lett. 12, 3151 (2012).

(27) M. Kumagai, and T. Takagahara, "Excitonic and nonlinear-optical properties of dielectric quantum-well structures", Phys. Rev. B 40, 12359 (1989).

(28) J. L. Movilla, J. I. Climente, and J. Planelles, "Dielectric polarization in axiallysymmetric nanostructures: A computational approach", Comp. Phys. Comm. 181, $92(2010)$.

(29) O. Millo, D. Katz, Y. W. Cao, and U. Banin, "Imaging and Spectroscopy of ArtificialAtom States in Core/Shell Nanocrystal Quantum Dots", Phys. Rev. Lett. 86, 5751 (2001). 
(30) The spectral feature at $2.38 \mathrm{eV}$ in Fig. 2(a) is a nearly degenerate doublet.

(31) F. Rajadell, J. I. Climente, J. Planelles, "Excitons in core-only, core-shell and corecrown CdSe nanplatelets: Interplay between in-plane electron-hole correlation, spatial confinement, and dielectric confinement", Phys. Rev. B 96, 035307 (2017).

(32) M. Richter, "Nanoplatelets as a material system between strong confinement and weak confinement", Phys. Rev. Materials 1, 016001 (2017).

(33) L. E. Brus, "A simple model for the ionization potential, electron affinity, and aqueous redox potentials of small semiconductor nanocrystallites", J. Chem. Phys. 79, 5566 (1983).

(34) M. A. Jazi, V. A. E. C. Janssen, W. H. Evers, A. Tadjine, C. Delerue, L. D. A. Siebbels, H. S. J. van der Zant, A. J. Houtepen, and D. Vanmaekelbergh, "Transport Properties of a Two-Dimensional PbSe Square Superstructure in an Electrolyte-Gated Transistor", Nano Lett. 17, 5238 (2017).

(35) A. Polovitsyn, Z. Dang, J.L. Movilla, B. Martín-García, G.H.V. Bertrand, R. Brescia, and I. Moreels, "Synthesis of Air-Stable CdSe/ZnS Core-Shell Nanoplatelets with Tunable Emission Wavelength", Chem. Mater 29, 5671 (2017).

(36) J. Yang, and F. W. Wise, "Electronic States of Lead-Salt Nanosheets", J. Phys. Chem. C 119, 26809 (2015).

(37) H. J. Krenner, M. Sabathil, E. C. Clark, A. Kress, D. Schuh, M. Bichler, G. Abstreiter, and J. J. Finley, "Direct Observation of Controlled Coupling in an Individual Quantum Dot Molecule", Phys. Rev. Lett. 94, 057402 (2005).

(38) G. Raino, M. A. Becker, M. I. Bodnarchuk, R. F. Mahrt, M. V. Kovalenko, T. Stöferle, "Superflourescence from lead halide perovskite quantum dot superlattices", Nature 563, 671 (2018). 
(39) D. Kim, S. Tomita, K. Ohshiro, T. Watanabe, T. Sakai, I.Y. Chang, and K. HyeonDeuk, "Evidence of Quantum Resonance in Periodically-Ordered Three-Dimensional Superlattice of CdTe Quantum Dots", Nano Lett. 15, 4343 (2015).

(40) R. Scott, A. V. Prudnikau, A. Antanovich, S. Christodoulou, T. Riedl, G. H. V. Bertrand, N. Owschimikow, J. K. N. Lindner, Z. Hens, I. Moreels, M. Artemyev, U. Woggon and A. Achtstein, "A comparative study demonstrates strong size tunability of carrier-phonon coupling in CdSe-based 2D and 0D nanocrystals" Nanoscale 11, 3958 (2019). 


\section{Graphical TOC Entry}

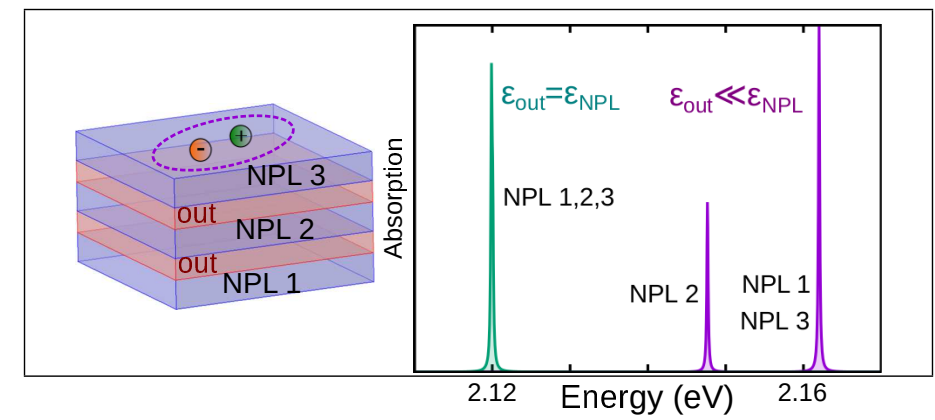




\title{
Supplemental Material for “Dielectric
}

\section{Confinement Enables Molecular Coupling in Stacked Colloidal Nanoplatelets"}

\author{
José L. Movilla, ${ }^{\dagger}$ Josep Planelles, ${ }^{\ddagger}$ and Juan I. Climente, ${ }^{*}$ \\ †Dept. d'Educació i Didàctiques Específiques, Universitat Jaume I, 12080, Castelló, Spain \\ $\ddagger$ Departament de Química Física i Analítica, Universitat Jaume I, E-12080, Castelló de la \\ Plana, Spain \\ E-mail: climente@uji.es
}

\section{Theoretical Method}

As mentioned in the main text, independent electrons and holes are described with effective mass Hamiltonians,

$$
H^{j}=\frac{\mathbf{p}_{\perp}{ }^{2}}{2 m_{\perp}^{j}}+\frac{p_{z}^{2}}{2 m_{z}^{j}}+V_{b o}^{j}\left(\mathbf{r}_{\mathbf{j}}\right)+\Sigma\left(\mathbf{r}_{\mathbf{j}}\right) .
$$

where $\mathbf{p}=-i \hbar \nabla_{\perp}$ and $\mathbf{p}_{z}=-i \hbar \nabla_{z}^{2}$ are the momentum operators parallel and perpendicular to the strong confinement direction of the NPL, $m_{z}^{j}\left(m_{\perp}^{j}\right)$ are the effective masses, $V_{b o}^{j}$ the potential band-offset between the materials that constitute the NPL-ligand system, $\Sigma$ the self-energy potential arising from the dielectric mismatch between NPL and organic environment, and $j=e, h$ denotes electron and hole, respectively. Material parameters are summarized in the table below. Dielectric constants of CdSe and CdTe are set equal for simplicity. Because dielectric mismatch effects scale proportional to $1 / \epsilon_{1}-1 / \epsilon_{2}$, and both materials have large dielectric constants, this is a reasonable simplification. 


\begin{tabular}{|c|c|c|c|c|c|c|}
\hline Description & Symbol & CdSe & CdTe & Ligand & Units & Refs. \\
\hline Electron mass $z$ & $m_{z}^{e}$ & 0.12 & 0.09 & 1.0 & $m_{0}$ & 1 \\
\hline Electron mass $\perp$ & $m_{\perp}^{e}$ & 0.12 & 0.09 & 1.0 & $m_{0}$ & 1 \\
\hline Hole mass $z$ & $m_{z}^{h}$ & 1.14 & 0.53 & 1.0 & $m_{0}$ & ${ }^{2}(\mathrm{CdSe}),{ }^{3}(\mathrm{CdTe})$ \\
\hline Hole mass $\perp$ & $m_{\perp}^{h}$ & 0.38 & 0.14 & 1.0 & $m_{0}$ & ${ }^{2}(\mathrm{CdSe}),{ }^{3}(\mathrm{CdTe})$ \\
\hline Conduction band offset & $V_{b o}^{e}$ & 0 & 0.53 & 2 & $\mathrm{eV}$ & $4(\mathrm{CdTe}),{ }^{5}$ (ligand) \\
\hline Valence band offset & $V_{b o}^{h}$ & 0 & -0.69 & 2 & $\mathrm{eV}$ & ${ }^{4}(\mathrm{CdTe}),{ }^{5}$ (ligand) \\
\hline Dielectric constant & $\epsilon$ & 10 & 10 & 2 & $\epsilon_{0}$ & 1 (CdSe, CdTe) ${ }^{6}$ (ligand) \\
\hline
\end{tabular}

where $m_{0}$ and $\epsilon_{0}$ are the free electron mass and vacuum permittivity, respectively.
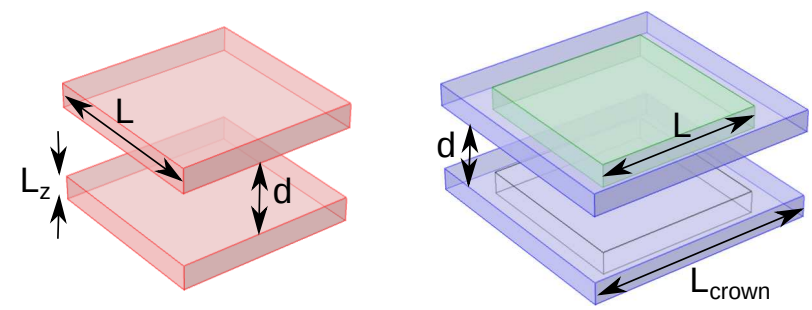

Figure S1: Geometry of the NPL structures under study. The example corresponds to a stack of 2 NPLs. Left: core-only. Right: core-crown NPLs.

For our simulations, we consider CdSe NPLs with 4.5 atomic monolayers thickness $\left(L_{z}=\right.$ $1.35 \mathrm{~nm}$ ). We consider the NPL to be square, with lateral side $L=10 \mathrm{~nm}$. These are close to standard experimental dimensions. ${ }^{7}$ One has to bear in mind that increasing the anistropy (larger lateral dimensions, thinner NPL) will further lift the compensation between selfenergy and Coulomb polarization terms, which will make the effects we predict even more conspicuous. In the case of core-crown NPLs, we add a CdTe crown surrounding the CdSe core, with total lateral side $L_{\text {crown }}=14 \mathrm{~nm}$. The edge-to-edge inter-NPL spacing is $d$. In experiments, this corresponds roughly to twice the capping ligand length (even though interdigitation may reduce this value). ${ }^{8}$ Fig. S1 illustrates the structures under study.

Usual analytical expressions to calculate $\Sigma$, based on the method of image charges, ${ }^{9}$ become impractical in stacks. This is in part due to the large number of dielectric interfaces, 
which make the expressions increasingly cumbersome, and especially to the pathological behavior of self-energy on the abrupt interfaces,${ }^{10}$ which prevents a physically sound description of possible quantum tunneling across stacked NPLs. We then calculate the surface induced charge using Ref. ${ }^{11}$ algorithms and codes assuming a dielectric interface of $0.4 \mathrm{~nm}$ thickness in between NPL and environment. More details and validation of these calculations are given in the next subsection.

Hamiltonian (1) is solved using Comsol Multiphysics 4.2. For computational efficiency, we limit our calculations to stacks of up to 5 NPLs. Because the redshift due to weakening of $\Sigma$ rapidly saturates with increasing number of NPLs, e.g. compare the 4 and 5 NPLs shifts in Fig. 1(b) of the main text, we expect this to be an approximate (lowerbound) value to that of a periodic array. Thus, in Fig.1(c) the mini band width, $\Delta_{\text {band }}$, is calculated as the energy difference between the ground and fifth states of a 5-NPL stack.

For excitonic systems, the Hamiltonian is:

$$
H_{X}=H_{e}+H_{h}+V_{e-h}
$$

where $H_{e}$ and $H_{h}$ are given by Eq. (1) and $V_{e-h}$ is the e-h Coulomb interaction. The latter is obtained by calculating the Poisson equation in a dielectrically inhomogeneous environment, using the electron and hole densities as source charges in Comsol Multiphysics. Notice Coulomb term is calculated in quantum boxes (like those in Fig. S1), while $\Sigma$ was calculated in an infinite well approximation. However, because lateral sides are much larger than the thickness $\left(L=10 \mathrm{~nm}\right.$ vs $\left.L_{z}=1.35 \mathrm{~nm}\right)$, most of the surface polarization takes place in the top and bottom sides of the NPL, and the lateral walls produce negligible influence.

We approximate the exciton wave function as $|X\rangle=\sum_{i j} c_{i j}|e\rangle_{i}|h\rangle_{j}$, where $|e\rangle_{i}$ and $|h\rangle_{j}$ are independent e-h states. The basis set is formed by all electron and hole spin-orbitals associated with $s, p_{x}$ and $p_{y}$ type (i.e. no nodes, one node along $x$, one node along $y$ ). Coulomb matrix elements are integrated numerically and the resulting projection of Hamiltonian (2) 
diagonalized.

\section{Self-energy potential in stacks of NPLs}

In brief, our model ${ }^{11}$ calculates numerically the surface polarization charges induced by the real charge at the finite-width dielectric transition layer separating the NPLs and the ligands. Afterwards, the self-polarization potential is calculated as the potential felt by the inducing charge due to its own surface-induced charges. The relatively low symmetry of the NPL system $\left(D_{4 h}\right)$ turns the evaluation of the self-energy potential into a rather demanding computational task, since the procedure goes through calculating the (threedimensional) distribution of the surface polarization charge for each point at which the potential is evaluated. Additionally, the computational cost growths rapidly with the number of dielectric interfaces (i.e., with the number of platelets in the stack). To obtain a tractable yet valid model, we replace the square-shaped NPLs by round-shaped disks, which increases the symmetry of the system to axial. Furthermore, we take advantage of the large anisotropy of NPLs to evaluate $\Sigma$ along the symmetry axis only, turning the 3D problem into a $2 \mathrm{D}$ one. The resulting profile of $\Sigma$ can then be extrapolated to the entire structure provided that the electron and hole density distributions near the lateral limits of the NPLs are negligible, and that the lateral interfaces exert a negligible influence on the obtained $\Sigma$ profile. That is, the profile is an approximation of the infinite quantum well limit.

Figure S2 confirms the validity of this approximation. The self-potential calculated along the symmetry axis of a single CdSe NPL is depicted for two different dielectric interface thicknesses $(\delta=0.5 \mathrm{~nm}$ and $\delta=0.3 \mathrm{~nm})$. For the sake of comparison, we also include the self-polarization potential corresponding to a colloidal quantum well with abrupt dielectric interfaces (dashed lines in the Figure), obtained by using the analytical expressions in Ref. ${ }^{9}$ As can be observed, the $\Sigma$ profile approaches that of the quantum well as $\delta$ is reduced (i.e.,

as the steepness of the transition layer between the different dielectric media increases), especially within the NPL. 


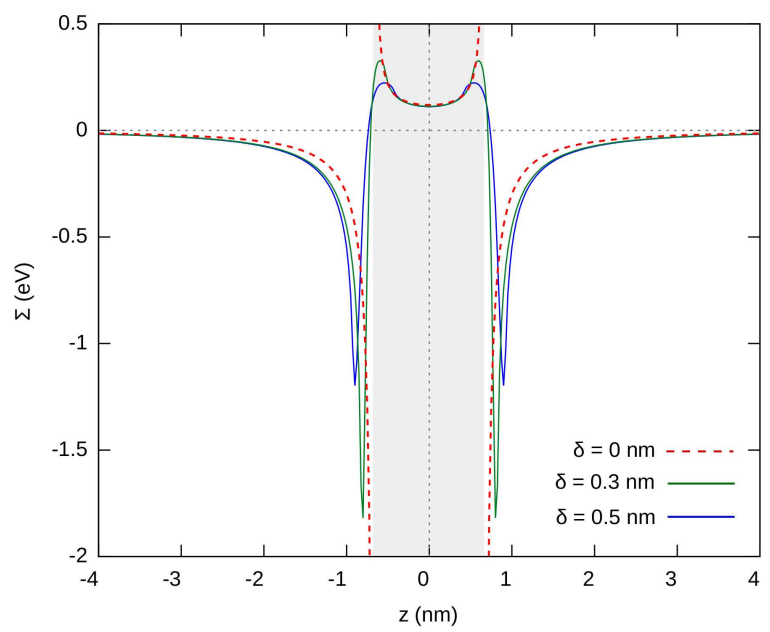

Figure S2: Self-energy potential along the strong confinement direction of a 4.5 monolayers thick CdSe NPL (shaded area). The solid profiles are obtained numerically assuming diskshaped NPLs with finite-width $(\delta=0.5 \mathrm{~nm}$ (blue) and delta $=0.3 \mathrm{~nm}$ (green)) dielectric interfaces. The red, dashed profile is obtained by using the analytical formulae for a quantum well with abrupt $(\delta=0)$ dielectric interfaces (see, e. g., Ref. $\left.{ }^{9}\right)$.

In stacks of NPLs, the self-energy of a charge carrier confined in a NPL can be expected to be affected by the presence of additional dielectric interfaces. Figures S3(a) and (b) compare $\Sigma$ along the $z$-axis in a system of two stacked NPLs separated by $d=2 \mathrm{~nm}$ with those calculated by artificially removing one or the other. Likewise, Figs. S3(c) and (d) compare the complete calculation of the system $\Sigma$ with that obtained by superimposing $\Sigma$ for the two independent NPLs. The comparisons evidence that (i) charges in stacked NPLs are more stabilized than in isolated NPLs, and (ii) $\Sigma$ is quasi-additive down to small interNPL distance values. These results allows us to discuss self-energy effects in terms of direct superimposition of $\Sigma$ of individual NPLs.

Further evidence on the stabilizing effect of nearby NPLs can be observed in Fig. S4, which represents $\Sigma$ for a stack of 5 NPLs. The bottom panel focuses on the self-potential profile within the NPLs. The figure reveals that internal NPLs are stabilized as compared to the terminal ones. The more internal the NPL, the larger the stabilization. This is a consequence of the weaker dielectric confinement, as discussed in the main text. 

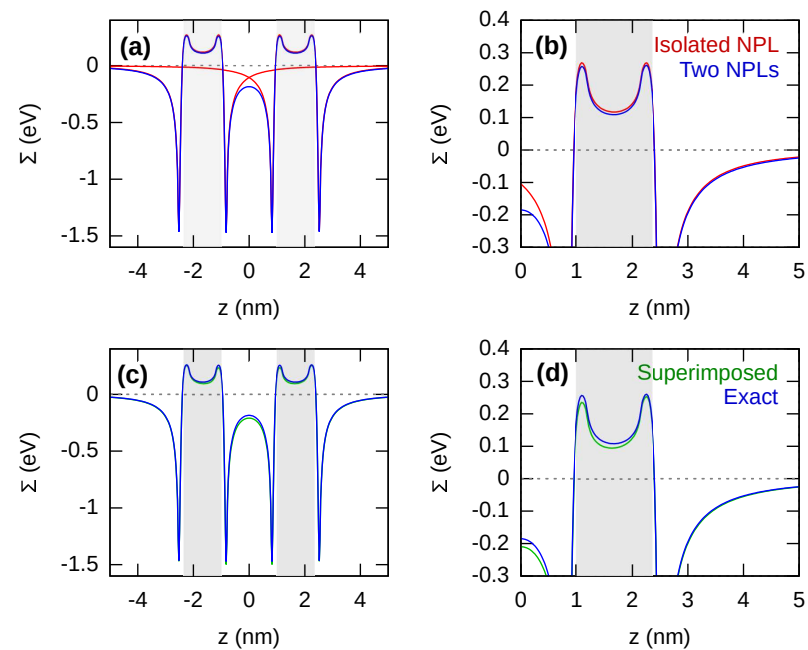

Figure S3: (a) and (b): Self-energy potential profiles for two stacked NPLs separated by $d=2 \mathrm{~nm}$ (blue lines) and those corresponding to each of the NPLs in the absence of the other (red lines). (c) and (d): Same but comparing $\Sigma$ of the 2 NPL stack with the sum of $\Sigma$ obtained for each of the two NPLs independently (green lines).

\section{Supporting Calculations}

In Fig. S5 we show the calculated optical absorption spectrum neglecting dielectric mismatch. That is, a homogeneous relative dielectric constant $\epsilon=10$ is taken all over the structure. In contrast to Fig. 3(c) of the main text, all the exciton states are now degenerate. This confirms the need of dielectric confinement terms to lower the system symmetry and display the rich exciton structure discussed in our work.

Fig. S6 illustrates the variation of the absorption spectrum of a pair of dielectrically equivalent NPLs as Coulomb interaction is switched on. As in Fig. 2(a) of the main text, for nearly non-interacting e-h pair (bottom curve) two peaks are observed split by quantum tunneling. These correspond to $|+\rangle_{e}|+\rangle_{h}$ and $|-\rangle_{e}|-\rangle_{h}$ states, where electron and hole localize in either NPL indistinctly. As e-h interaction increases, the states evolve into direct and indirect excitons (see main text). The latter becomes dark and energetically less stable because of the spatial separation of electron and hole.

Fig. S7 shows the variation of Coulomb tunneling term $K$ as a function of the inter-NPL 

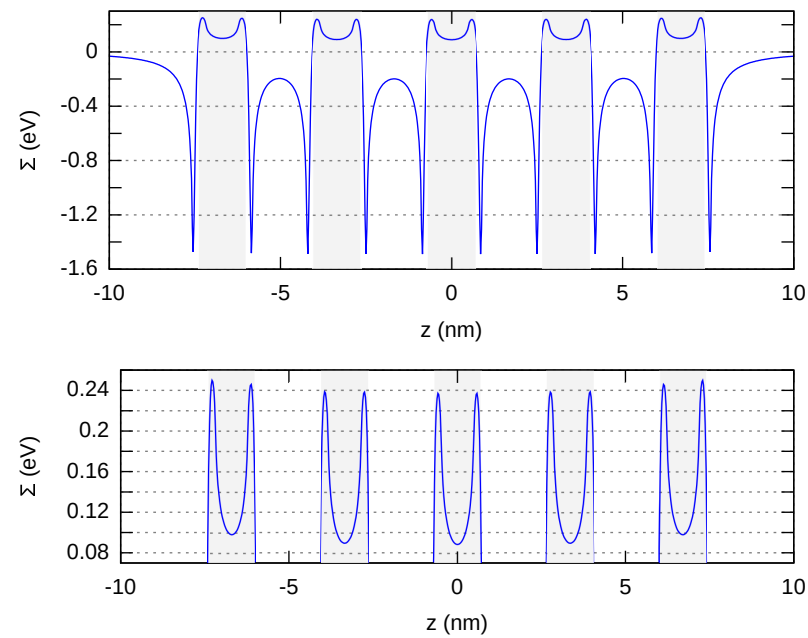

Figure S4: Self-energy potential for a stack comprised of five CdSe NPLs separated by $d=$ $2 \mathrm{~nm}$. The bottom panel evidences the less repulsive character of $\Sigma$ in the inner NPLs, as compared to terminal NPLs.

distance $d$. The closer the NPLs, the smaller the Coulomb tunneling. This is because indirect excitons start feeling Coulomb attraction as the NPLs are approached, which reduces the energy gap with respect to direct excitons. Nevertheless, $K$ is large (40 meV) even for even for $d=1 \mathrm{~nm}$. The large value and the high sensitivity is due to the low dielectric screening of the inter-NPL buffer layer $\left(\epsilon_{\text {out }}=2\right)$, which is a differential feature as compared to coupled epitaxial heterostructures.

Fig. S8 shows the different charge density localization in a 3 NPL stack, for ground and first optically active state. As indicated in the main text, the ground state of electrons and excitons alike localize in the central NPL, where $\Sigma$ is smallest, and the excited state in the terminal NPLs. 


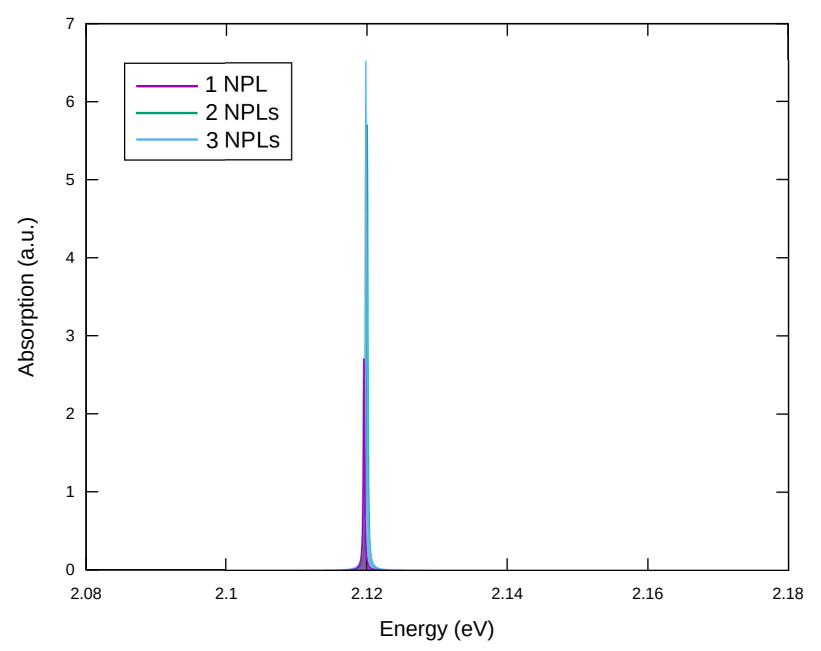

Figure S5: Exciton absorption spectrum in stacks of 1 to 3 NPLs, disregarding dielectric mismatch (i.e. same dielectric constant in NPLs and in inter-NPL layers). Inter-NPL distance is $d=3 \mathrm{~nm}$.

\section{References}

(1) O. Madelung, Semiconductors: Data Handbook, (Springer-Verlag, Berlin, 2004).

(2) D. J. Norris, and M. G. Bawendi, "Measurement and assignment of the size-dependent optical spectrum in CdSe quantum dots", Phys. Rev. B 53, 16338 (1996).

(3) S. Adachi, Handbook on Physical Properties of Semiconductors, volume 3, (Kluwer Academic Pub., Boston, 2004).

(4) Y. H. Li, A. Walsh, S. Chen, W. J. Yin, J. H. Yang, J. Li, J. L. F. Da Silva, X. G. Gong, and S. H. Wei, "Revised ab initio natural band offsets of all group IV, II-VI, and III-V semiconductors", Appl. Phys. Lett. 94, 212109 (2009).

(5) A. Chu, C. Livache, S. Ithurria, and E. Lhuillier, "Electronic structure robustness and design rules for 2D colloidal heterostructures", J. Appl. Phys. 123, 035701 (2018).

(6) A.W. Achtstein, A. Schliwa, A. Prudnikau, M. Hardzei, M.V. Artemyev, C. Thomsen, and U. Woggon, "Electronic Structure and Exciton-Phonon Interaction in TwoDimensional Colloidal CdSe Nanosheets", Nano Lett. 12, 3151 (2012). 


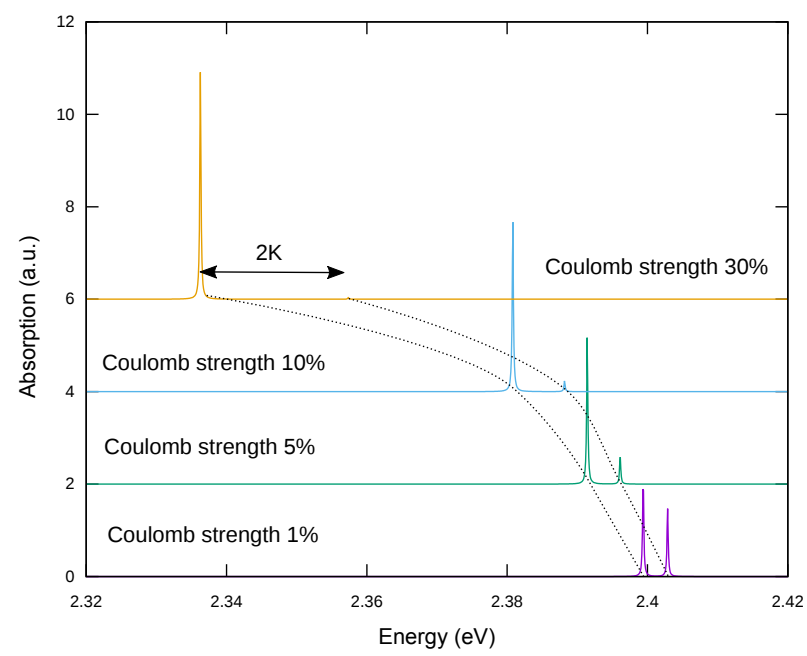

Figure S6: Exciton absorption spectrum in a stack of two closely spaced $(d=1 \mathrm{~nm})$ NPLs, with varying strength of e-h Coulomb interaction.

(7) G. H. V. Bertrand, A. Polovitsyn, S. Christodoulou, A. H. Khan, and I. Moreels. "Shape control of zincblende CdSe nanoplatelets." Chem. Commun. 52, 11975 (2016).

(8) S. Jana, T. N. T. Phan, C. Bouet, M. D. Tessier, P. Davidson, B. Dubertret, and B. Abecassis, "Stacking and Colloidal Stability of CdSe Nanoplatelets", Langmuir 31, $10532(2015)$.

(9) M. Kumagai, and T. Takagahara, "Excitonic and nonlinear-optical properties of dielectric quantum-well structures", Phys. Rev. B 40, 12359 (1989).

(10) L. Banyai, S. W. Koch, Semiconductor quantum dots, (World Scientific, Singapore, 1993).

(11) J. L. Movilla, J. I. Climente, and J. Planelles, "Dielectric polarization in axiallysymmetric nanostructures: A computational approach", Comp. Phys. Comm. 181, $92(2010)$ 


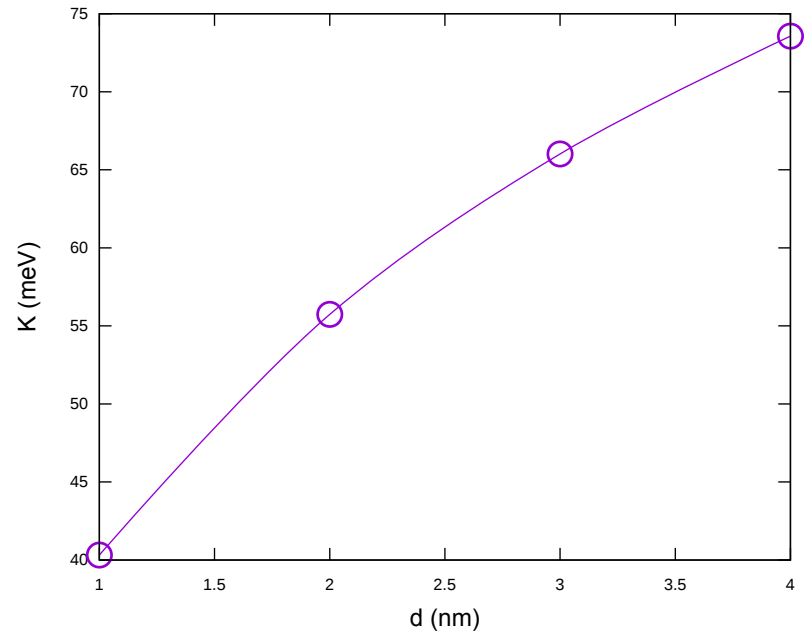

Figure S7: Coulomb tunneling term $K$ as a function of the inter-NPL distance in a stack of 2 NPLs.

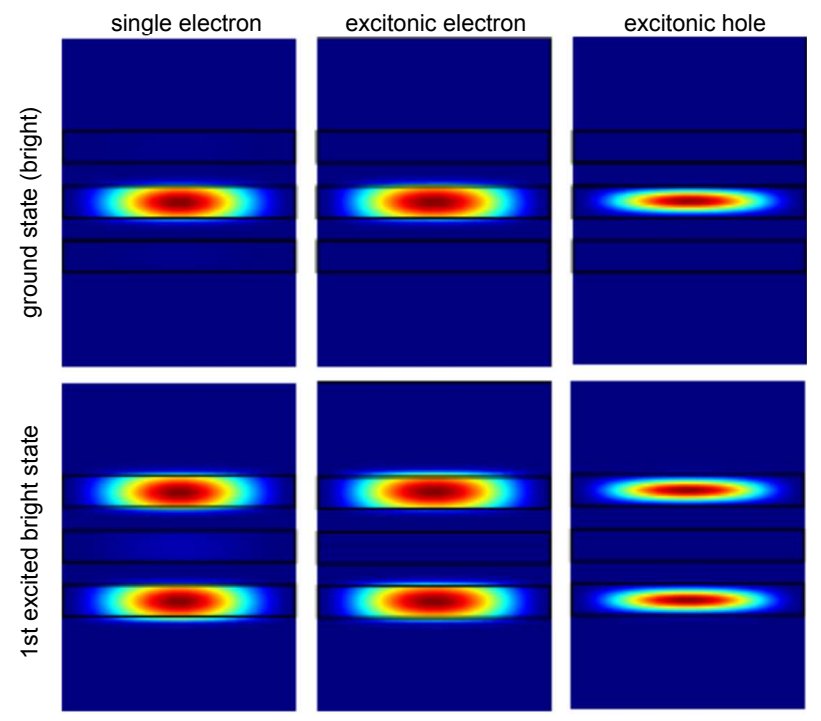

Figure S8: Left: charge density of single electron ground and first excited state in a 3 NPL stack. Center: same but for excitonic electron inside an exciton. Right: same but for excitonic hole. 